\title{
Orientaciones Prácticas para Psicoterapeutas que Atienden a Pacientes con TEPT Después de un Desastre Natural
}

\section{Practical Guidelines for Psychotherapists Who Work With PTSD Patients After a Natural Disaster}

\author{
Paula Errázuriz, Candice Fischer, Alex Behn, Catalina Letelier y Marietta Monari \\ Pontificia Universidad Católica de Chile
}

\begin{abstract}
Este artículo presenta información relevante para profesionales de la salud mental que trabajarán con pacientes adultos con trastorno por estrés post-traumático (TEPT) en las postrimerías de un desastre de origen natural y que no poseen entrenamiento especializado en modalidades expertas para el manejo de TEPT. El artículo presenta orientaciones a partir de una revisión de diferentes guías internacionales para el trabajo con pacientes con TEPT y la revisión de la literatura científica internacional relevante. Estas orientaciones fueron revisadas por un panel de expertos chilenos. El resultado, el cual se presenta en este artículo, es una serie de orientaciones prácticas orientadas a aquellos psicoterapeutas que trabajarán con pacientes con TEPT tras la ocurrencia de un desastre de origen natural. Se presentan viñetas clínicas para ejemplificar algunas de las orientaciones.
\end{abstract}

Palabras clave: desastres de origen natural, estrés post-traumático, psicoterapia, orientaciones

\begin{abstract}
This article presents information relevant to mental health professionals who work with adult patients with posttraumatic stress disorder (PTSD) in the aftermath of natural disasters and who lack specialized training in evidencebased treatments for PTSD. The article presents orientations based on a review of several international guidelines for working with patients with PTSD and the relevant international scientific literature. These guidelines were reviewed by a panel of Chilean experts. The result, which is presented in this article, consists in a series of practical guidelines aimed at psychotherapists who must work with patients with PTSD after natural disasters. Clinical vignettes are presented to exemplify some of these guidelines.
\end{abstract}

Keywords: natural disasters, post-traumatic stress, psychotherapy, guidelines

Los desastres de origen natural y sus consecuencias físicas y mentales han cobrado relevancia en la agenda de los sistemas de salud de varios países, tales como Estados Unidos, Reino Unido y Australia, siendo reconocido como un problema de salud pública de alta proporción (Australian Center for Posttraumatic Mental Health [ACPMH], 2013; Cook \& Newman, 2014; U.S. Department of Health and Human Services, 2003). Para orientar la acción de los profesionales de la salud, estos países han diseñado manuales, que, basándose en evidencia científica, ofrecen recomendaciones prácticas sobre cómo actuar en el contexto de un desastre natural. Sin embargo, la mayoría de estos manuales, incluyendo el Manual para la Protección y Cuidado de la Salud Mental en Situaciones de Emergencias y Desastres del Ministerio de Salud de Chile (MINSAL, 2012), se centran en brindar indicaciones de primeros auxilios psicológicos y pocos de ellos desarrollan el manejo y la atención en psicoterapia de las víctimas de trastorno por estrés post-traumático (TEPT) y, de los que lo desarrollan, la gran mayoría no está en español. Una excepción a lo anterior es el trabajo de Encinas y Valdés (2012), quienes adaptaron la terapia cognitivo- conductual para estrés postdesastre desarrollada en Estados Unidos por Hamblen, Gibson, Mueser y Norris (2006), usándola para atender a víctimas del terremoto del 27 de febrero de 2010 en Chile.

Paula Errázuriz, Candice Fischer y Catalina Letelier, Escuela de Psicología, Pontificia Universidad Católica de Chile, y National Research Center for Integrated Natural Disaster Management, Santiago, Chile; Alex Behn y Marietta Monari, Escuela de Psicología, Pontificia Universidad Católica de Chile, Santiago, Chile; Paula Errázuriz, Candice Fischer y Alex Behn, Instituto Milenio para la Investigación en Depresión y Personalidad.

Este estudio recibió apoyo económico de la Comisión Nacional de Investigación Científica y Tecnológica de Chile a través del proyecto FONDAP 15110017 y del Fondo de Innovación para la Competitividad (FIC) del Ministerio de Economía, Fomento y Turismo, a través de la Iniciativa Científica Milenio, Proyecto IS130005. Los autores agradecen especialmente el trabajo de Catalina Núñez y Stephanie Vaccarezza como asistentes de investigación.

La correspondencia relativa a este artículo debe ser dirigida a Alex Behn, Escuela de Psicología, Pontificia Universidad Católica de Chile, Avda. Vicuña Mackenna 4860, Macul, Santiago, Chile. E-mail: albehn@uc.cl 
Los tratamientos para TEPT son altamente especializados y, por lo general, desplegados por profesionales con entrenamiento específico. Sin embargo, en las postrimerías de un desastre de origen natural se espera que aumenten los pacientes con TEPT, aumento que en Chile sobrepasaría con creces la disponibilidad de profesionales específicamente entrenados para tratar este trastorno. Tras un desastre de origen natural, muchos psicoterapeutas se encontrarán, de hecho, trabajando con pacientes con TEPT sin poseer el entrenamiento necesario, con lo cual se hace imprescindible el desarrollo de una guía práctica que sistematice los estándares internacionales para el tratamiento del TEPT. Debido a lo anterior, el objetivo de este artículo es presentar información en español útil para psicólogos que, sin contar con formación especializada para ello, trabajan con pacientes adultos que sufren TEPT luego de un desastre de origen natural en Chile. Este artículo no reemplaza la formación especializada para el tratamiento del TEPT, la cual es necesaria para trabajar adecuadamente con esta población de pacientes. Lo que en este artículo sí se ofrece son orientaciones generales para el tratamiento del TEPT que esperamos puedan servir de introducción al tema y ser una guía para el profesional que, debido a una emergencia, se encuentra trabajando con pacientes con TEPT sin la formación necesaria.

Describiremos brevemente lo que es un desastre de origen natural, el TEPT, su prevalencia y factores de riesgo. Luego presentaremos el método que usamos para elegir y adaptar las orientaciones propuestas, las cuales son finalmente presentadas en la sección de Orientaciones Prácticas.

\section{¿Qué es un Desastre de Origen Natural?}

Cuando un evento de origen natural (e.g., un terremoto, tsunami o aluvión) produce una "interrupción seria del funcionamiento de una comunidad o sociedad que causa pérdidas humanas y/o importantes pérdidas materiales, económicas o ambientales; [y] éstas exceden la capacidad de la comunidad o sociedad afectada para hacer frente a la situación, utilizando sus propios recursos" (Organización de las Naciones Unidas, 2004, p. 17) estamos en presencia de un desastre de origen natural.

Chile es históricamente un país afectado por una amplia diversidad de desastres de origen natural, incluyendo grandes terremotos, tsunamis, erupciones volcánicas y aluviones. Recientemente hemos sido testigos nuevamente de la vulnerabilidad que afecta a nuestro país, con los incendios en las regiones del Bíobío, La Araucanía, Los Ríos y Los Lagos y el incremento de caudales de los ríos y aluviones en las regiones de Tarapacá, Antofagasta, Atacama y Coquimbo (Centro Sismológico Nacional Universidad de Chile, 2016; MINSAL, 2010; Chile, Oficina Nacional de Emergencia del Ministerio del Interior y Seguridad Pública, 2018).

\section{¿Qué Tipo de Respuestas Psicológicas se Pueden Esperar en los Afectados por Desastres de Origen Natural?}

El tipo de respuesta específico de las personas expuestas a un desastre de origen natural dependerá del tipo, intensidad y duración del desastre, características personales y factores sociales, entre otros elementos, por lo que las reacciones a los desastres y la severidad de los síntomas varían ampliamente (Bonanno, Galea, Bucciarelli \& Vlahov, 2007; Garfin, Silver, Ugalde, Linn \& Inostroza, 2014; Ozer, Best, Lipsey \& Weiss, 2003). Sin embargo, no todas las víctimas de un desastre natural desarrollarán un trastorno en salud mental y, en general, la mayoría de los afectados presentará síntomas psicológicos transitorios que remitirán a corto plazo con apoyo psicosocial y práctico, sin presentar posteriores dificultades en su salud mental (Courtois \& Gold, 2009; Neria, Nandi \& Galea, 2008). Bonanno et al. (2007) estimaron que hasta un $65 \%$ de sujetos expuestos en diferentes grados a eventos potencialmente traumáticos siguen trayectorias de resiliencia y no desarrollarán TEPT. Entre aquellos sujetos con exposición directa a eventos potencialmente traumáticos, se estima que un $25 \%$ sí desarrollará TEPT. Este subgrupo de personas requerirá de intervenciones altamente especializadas o, en su defecto, intervenciones informadas razonablemente en la evidencia empírica que tiendan a maximizar las probabilidades de recuperación de los pacientes y a minimizar los posibles efectos iatrogénicos de aplicaciones parciales o incompletas de los tratamientos especializados especificados para TEPT.

\section{¿Qué Es el Trastorno por Estrés Post-Traumático?}

El TEPT se caracteriza por la presencia de la siguiente sintomatología, luego de experimentar un suceso excepcionalmente angustiante, que amenaza la vida del individuo o de otras personas: evocaciones o representaciones del acontecimiento en forma de recuerdos o imágenes durante la vigilia o de ensueños 
reiterados; un malestar psicológico intenso o prolongado con evitación de los estímulos asociados al evento traumático; y alteraciones negativas cognitivas, del estado de ánimo, del sueño, de la alerta y de la reactividad, de una duración superior a un mes (American Psychiatric Association, 2013; Organización Mundial de la Salud [OMS], 1992). Cuando la duración de los síntomas va de los tres días a un mes, el diagnóstico es de un trastorno de estrés agudo, el que puede ser cambiado a TEPT si supera el mes de duración. A su vez, en el caso de que la totalidad de los criterios diagnósticos para TEPT no se cumplan hasta al menos seis meses después del evento traumático, se puede diagnosticar TEPT con expresión retardada. En la Tabla 1 se pueden revisar todos los criterios diagnósticos para TEPT propuestos por la Clasificación Internacional de Enfermedades, $10^{a}$ versión (CIE 10; OMS, 1992).

Tabla 1

Criterios Diagnósticos de TEPT Según la CIE-10

A. El paciente ha estado expuesto a un acontecimiento estresante o a una situación (breve o duradera) de naturaleza excepcionalmente amenazante o catastrófica, que causarían por sí mismos malestar generalizado en casi todo el mundo.

B. Se dan episodios reiterados de revivir el trauma en forma de flashbacks, recuerdos vívidos o sueños recurrentes, o que consisten en experimentar malestar cuando la persona se ve expuesta a situaciones que se asocian o se parecen a la vivida.

C. Suelen temerse, e incluso evitarse, las situaciones que recuerdan o sugieren el trauma vivido, temor que no existía antes de la exposición al hecho traumático.

D. Deben satisfacerse alguno de los siguientes síntomas:

1. Incapacidad para recordar, ya sea parcial o totalmente, algunos aspectos importantes del período de exposición al hecho estresante.

2. Síntomas persistentes de incremento de sensibilidad psicológica y excitación (no presentes antes de la exposición al hecho estresante), que se manifiestan con dos de las siguientes características:

a. dificultad para dormir o permanecer dormido (insomnio)

b. irritabilidad o arranques de ira

c. dificultad para concentrarse

d. hipervigilancia

e. sobresaltos exagerados

E. Los criterios B, C y D deben haberse dado en los 6 meses posteriores al episodio vivido o al final del período de estrés (en algunos casos y con determinados objetivos, puede incluirse en el diagnóstico un caso en que la aparición del trastorno se produce cuando han transcurrido más de 6 meses, pero esto debe quedar claramente especificado).

Nota. Reimpreso de OMS (1992).

Existe una gran heterogeneidad en las presentaciones de TEPT (Galatzer-Levy \& Bryant, 2013), lo cual subraya la importancia del juicio clínico y conocimiento detallado de los criterios, así como de la patología nuclear del síndrome (Kelly, Rizvi, Monson \& Resick, 2009): (a) síntomas de activación fisiológica (e.g., hipervigilancia, irritabilidad, sobresalto, insomnio), (b) síntomas de re-experimentación (e.g., flashbacks, pesadillas, pensamientos intrusivos) y (c) síntomas de evitación (e.g., amnesia sobre detalles del trauma, reducción acusada del interés en actividades significativas, aislamiento, apropositividad). En la Tabla 2 exponemos la primera viñeta, en la que relatamos los síntomas que presenta un paciente ficticio y hacemos referencia a los criterios diagnóstico que cumple del CIE 10.

Los trastornos comórbidos con TEPT más comunes son la depresión, abuso de sustancias y alcohol, y otros trastornos de ansiedad, como agorafobia, fobias simples y fobia social (Kessler, Sonnega, Bromet, Hughes \& Nelson, 1995; Kessler et al., 2005). Estudios muestran que cerca del 80\% de los individuos con TEPT reúne criterios para uno o más trastornos comórbidos (Breslau, Davis, Andreski \& Peterson, 1991; Kessler et al., 1995; Kessler et al., 2005). 
Tabla 2

Viñeta 1: Diagnóstico de TEPT

Sandra es una paciente ficticia de 22 años que consulta indicando que "vengo porque no me he sentido muy bien; llevo un tiempo que me siento como cansada, estresada, no sé". Relata que hace dos meses (Criterio E) se encontraba trabajando de guardaparque en una zona afectada por un incendio forestal. Sandra, al quedar atrapada, resultó con quemaduras de gravedad en parte de sus manos y espalda (Criterio A) y señala que "no puedo sacármelo de la cabeza y eso hace que me sienta como depre, decaída, sin ganas".

A su vez, Sandra reporta altos niveles de ansiedad: "me asusto por todos los ruidos, estoy muy alerta a todo y eso hace que esté irritable y nerviosa. Quizás es por eso o porque estoy con problemas para dormir, me despierto muchas veces durante la noche y eso hace que esté más cansada y enojona” (Criterio D).

Así, Sandra indica que cuando despierta amanece nerviosa. "Llevo harto tiempo soñando con el incendio, con estar atrapada, ver a mis compañeros aterrados, con quemaduras, asustada; y, cuando me despierto, me acuerdo de todo y no puedo parar de pensar en qué podría haber hecho para que no pasara” (Criterio B).

$\mathrm{Al}$ ir finalizando la sesión, Sandra señala encontrarse bastante preocupada, ya que indica que no está asistiendo al trabajo "porque ahí veo a mis amigos y me acuerdo de todo lo que pasó. Y, más encima, tengo que volver a ese lugar y eso me recuerda todo y prefiero no ir" (Criterio C).

\section{Prevalencia del TEPT}

A nivel mundial, la prevalencia del TEPT luego de desastres de origen natural varía de 30 a $40 \%$ en las víctimas directas, 10 a $20 \%$ entre los voluntarios y rescatistas y entre un 10 a $20 \%$ en la población general (Goldman \& Galea, 2014). La prevalencia de vida en Chile de TEPT en general es de 4,4\% (6,2\% en mujeres y 2,5\% en hombres; Zlotnick et al., 2006) y esta prevalencia tiende a aumentar tras la ocurrencia de desastres de origen natural. Según el Ministerio de Planificación de Chile (2010; actualmente Ministerio de Desarrollo Social), la prevalencia de sintomatología de TEPT luego del terremoto y tsunami del 2010 fue de $12 \%$ en las regiones afectadas, aumentando a 20-25\% en las regiones más afectadas por ambos desastres, y siendo mayor en mujeres y en personas de bajos ingresos. Estas cifras son similares a las reportadas por Leiva-Bianchi y Araneda (2013), quienes encontraron una prevalencia de TEPT de $22 \%$ en la población de tres de las zonas más afectadas por el terremoto del 2010 en Chile.

\section{Factores de Riesgo}

Además de la exposición al desastre, múltiples estudios han identificado una serie de factores de riesgo que están asociados al aumento de la prevalencia del TEPT. Estos estudios sugieren que es probable que en los grupos de alto riesgo el impacto del trauma se mantenga o aumente, a diferencia de la población general, en la cual se espera que no desarrollen TEPT (Neria et al., 2008).

Tienen un mayor riesgo de padecer TEPT luego de un desastre natural los sujetos que tuvieron una mayor proximidad física al desastre y los que perdieron seres cercanos y elementos materiales de envergadura (Leiva-Bianchi \& Araneda, 2013). También tienen mayor riesgo de TEPT las personas que cumplen con alguna o varias de las siguientes características: sexo femenino (Kessler et al., 1995), haber experimentado previamente acontecimientos traumáticos, presentar problemas psicológicos previos al estresor traumático, tener una historia familiar de psicopatología, experimentar percepción de amenaza de vida durante el trauma, no contar con soporte social posterior al evento y tener bajos ingresos o bajo apoyo social (Neria et al., 2008). Además, la presencia de respuestas emocionales negativas durante o inmediatamente después del suceso y la disociación peri traumática aumentan el riesgo (Franklin, Sheeran \& Zimmerman, 2002; Goldmann \& Galea, 2014; Ozer et al., 2003). 


\section{Método}

El proceso de elaboración de las orientaciones que presentamos en esta guía consistió en los siguientes pasos:

1. Revisión no sistemática de pautas, guías y manuales internacionales de buenas prácticas para el tratamiento del TEPT elaboradas por las siguientes agencias: ACPMH, 2013, International Society for Traumatic Stress Studies (Foa, Keane, Friedman \& Cohen, 2009), Inter-Agency Standing Committee (2007), U.S. Department of Veterans Affairs y U.S. Department of Defense (2010) y World Health Organization, War Trauma Foundation y World Vision International (2011). Revisamos todas estas guías para elaborar las orientaciones incluidas en este artículo. Sin embargo, debido a que la gran mayoría de las guías de buenas prácticas disponibles se encuentran desactualizadas y sus recomendaciones son similares (Forbes et al., 2010), dimos especial énfasis a la Australian Guidelines for the Treatment of Acute Stress Disorder \& Posttraumatic Stress Disorder (ACPMH, 2013) por ser la más reciente. Debido a que la gran mayoría de las recomendaciones vienen de esta fuente, no hemos repetido la referencia cada vez en el texto. A su vez, nuestro equipo de autores, que está conformado por investigadores que además son psicoterapeutas, consideró que esta guía era adecuada en términos clínicos para recoger recomendaciones útiles para nuestra realidad nacional. Por ello, tradujimos y resumimos las recomendaciones de intervención individual pertinentes contenidas en esta guía y las complementamos con información contenida en las otras guías señaladas. Es preciso que advirtamos que, según la guía ACPMH (2013) para el tratamiento de pacientes con TEPT, las intervenciones grupales pueden complementar, pero no deben reemplazar, la psicoterapia individual. Es importante señalar que la guía de ACPMH (2013) fue realizada a partir de una revisión sistemática de la literatura. Todas las recomendaciones que extrajimos de esta guía cuentan con el mayor nivel de evidencia científica. Sin embargo, es importante considerar que esta evidencia no es chilena. Si bien, como explicamos a continuación, las orientaciones fueron revisadas por un panel de expertos chilenos, se necesita investigación nacional para saber cuáles son las orientaciones más adecuadas a nuestra realidad.

2. Revisión de la literatura científica. La búsqueda de literatura la realizamos en las bases de datos PsycINFO, Scielo, PubMed, ProQuest y The Cochrane Database of Systematic Reviews. También revisamos publicaciones chilenas que no se encontraban indexadas. Las palabras clave (en inglés y español) utilizadas para la búsqueda electrónica fueron psicoterapia, desastres naturales, trastorno por estrés post traumático y TEPT. Los limitadores de selección fueron revisiones sistemáticas, meta-análisis, población adulta y publicaciones entre los años 2010 a 2014. El número de artículos se amplió mediante la identificación de estudios citados en la bibliografía revisada.

3. Desarrollo de orientaciones. Desarrollamos una propuesta de orientaciones en español basada en las pautas internacionales de intervención (en particular, ACPMH, 2013) y la complementamos con información encontrada en la revisión de la literatura científica.

4. Revisión de las orientaciones. Este último paso consistió en la revisión, crítica y complementación de las orientaciones por un panel de expertos (ver Tabla 3), con el fin de adaptar las orientaciones para la realidad chilena. Cada miembro del panel de expertos recibió una copia de las orientaciones y un cuestionario con preguntas abiertas y cerradas para poder evaluar cada orientación y aportar sugerencias. Las orientaciones finales incluyen elementos del ACPMH, la revisión de literatura científica y las sugerencias de los expertos. 
Tabla 3

Perfil de los Expertos Consultados

\begin{abstract}
Dr. Rodrigo Figueroa
Médico-cirujano y especialista en psiquiatría de adultos de la Pontificia Universidad Católica de Chile.

Representante de la Subsecretaría de Salud Pública en el Comité de Emergencias y Desastres del Ministerio de Salud, Gobierno de Chile, e Investigador Asociado del Centro Nacional de Investigación para la Gestión Integrada de Desastres Naturales. Es socio director del Centro de Intervención en Trauma, Estrés y Desastres y docente de la Escuela de Psicología de la Pontificia Universidad Católica de Chile.

Miembro de la Asociación Chilena de Estrés Traumático, Asociación de Psicoterapeutas EMDR Chile y de la Sociedad Chilena de Medicina Pre-hospitalaria y de Desastres.

Humberto Marin, PhD.

Psicólogo de la Universidad Diego Portales, Máster en Seguridad, Emergencias y Desastres y Doctor en Psicología Social de la Facultad de Medicina de la Universidad Complutense de Madrid.

Fundador de la Sociedad Chilena de Psicología en Emergencias y Desastres. Actualmente es socio director del Centro de Intervención en Trauma, Estrés y Desastres e Investigador Asociado del Centro Nacional de Investigación para la Gestión Integrada de Desastres Naturales.

Lydia Gómez Pérez, PhD.

Doctora en Psicología y Máster en Psicología de la Salud por la Universidad de Málaga España.

Investigadora y docente en la Escuela de Psicología de la Pontificia Universidad Católica de Chile. Su investigación se centra en examinar los predictores psicosociales del trastorno de estrés postraumático y su comorbilidad con los problemas de salud física en general y los síndromes de dolor crónico en particular.
\end{abstract}

\title{
Orientaciones Prácticas
}

El tratamiento de primera línea para pacientes con TEPT como secuela de un desastre de origen natural es la psicoterapia focalizada en el trauma, de la cual existen diferentes modalidades. Ninguna de estas terapias debe ser utilizada antes del establecimiento de un diagnóstico fundamentado de TEPT, el cual por definición no puede ser establecido antes de un mes tras al desastre de origen natural. Además, los tratamientos de primera línea para pacientes que desarrollan TEPT son altamente especializados y requieren entrenamiento específico. La orientación, por lo tanto, es - siempre que sea posible- derivar a estos pacientes a atención experta especializada. Sin embargo, al menos en Chile, las oportunidades para la formación, diseminación y derivación de estos tratamientos especializados son escasas y muchos psicólogos sin la formación experta se encontrarán, de hecho, atendiendo a pacientes con TEPT tras la ocurrencia de desastres naturales. Esto implica la necesidad de flexibilizar el despliegue de tratamientos para TEPT a condición de diseminar criterios básicos de indicación, tratamiento y protección del paciente respecto de potenciales efectos iatrogénicos de la implementación parcial o inexperta de tratamientos especializados.

\section{Tratamientos Recomendados Para Pacientes con TEPT}

En nuestro país, la terapia cognitivo-conductual para el estrés post-desastre (Hamblen et al., 2009) mostró ser efectiva en el tratamiento de personas con estrés luego de un desastre natural (Leiva-Bianchi, Cornejo, Fresno, Rojas \& Serrano, 2018). A nivel internacional, las intervenciones focalizadas en el trauma que cuentan con el mayor respaldo empírico para el tratamiento de TEPT son la terapia cognitivo-conductual (reestructuración cognitiva; Resick, Nishith, Weaver, Astin \& Feuer, 2002), la terapia por exposición prolongada (PE; Foa, Hembree \& Rothbaum, 2007) y la terapia por desensibilización y reprocesamiento por el movimiento ocular (EMDR; Shapiro,1989, 1995). Son estos los tratamientos que, de acuerdo a la evidencia científica internacional, deben ser considerados como tratamientos de primera línea y las orientaciones que hemos generado se basan en ellos. Es importante señalar que algunas intervenciones que se usan para tratar trauma no han sido incluidas dentro de las orientaciones, porque aún no existe suficiente evidencia empírica 
(e.g., terapia psicodinámica breve) o porque han sido testeadas y han resultado ser menos efectivas que las intervenciones recomendadas (e.g., terapia de hipnosis y orientación de apoyo).

\section{¿Cuáles Son los Objetivos Centrales de las Terapias Enfocadas en el Trauma?}

1. Exponer sistemáticamente al paciente a las memorias del trauma en un ambiente seguro, con el fin de producir habituación a la respuesta emocional y angustiosa. Esto indica al paciente que sus respuestas emocionales intensas están en relación a riesgos que objetivamente ya no existen. Es importante tener presente que esta técnica de exposición puede ser iatrogénica si no es realizada adecuadamente. Por esto, se recomienda un entrenamiento especializado previo a ser usada y, de no ser posible, leer más sobre el tema y aprender de colegas que manejen adecuadamente la técnica.

2. Evaluar las memorias traumáticas y el desarrollo de distorsiones cognitivas, con especial referencia a la relación entre el material de estímulo (lo visto, lo escuchado y las sensaciones que surgieron, entre otros) y los componentes de la respuesta (reacciones físicas y problemas en la adaptación, entre otros). Esto ayuda al paciente a modificar la relación entre la respuesta afectiva y los pensamientos que contribuyen a su mantenimiento y exacerbación.

3. Eliminar sistemáticamente las conductas de evitación (e.g., supresión, consumo de sustancias y distracciones) que contribuyen al desarrollo y mantenimiento de la sintomatología.

\section{¿Cuáles Son las Orientaciones Para Lograr Estos Objetivos?}

Para lograr adecuadamente los objetivos de exponer al paciente a las memorias de trauma, modificar la respuesta afectiva y cognitiva frente a estas memorias y eliminar las conductas de evitación, se sugiere:

- Que el paciente pueda recordar y pensar sobre las memorias traumáticas hasta que estas no creen niveles altos de malestar. En el caso de las técnicas de exposición es muy importante abstenerse de utilizar técnicas de relajación, ya que estas interrumpen la exposición y, por lo tanto, atrasan y obstaculizan la habituación.

- Identificar, desafiar y modificar sesgos, creencias o distorsiones de los pensamientos y memorias de la experiencia traumática. Se pueden utilizar técnicas como el diálogo socrático y tareas para la casa en la forma de registro de pensamientos automáticos relacionados con el trauma. Se deben examinar las conductas de evitación (e.g., "no voy a ir al trabajo porque puede temblar de nuevo"), ya que frecuentemente se encuentran anudadas a distorsiones cognitivas y contribuyen a la mantención del TEPT, por lo cual deben ser examinadas y desmontadas.

- Ayudar a manejar los sentimientos que emerjan al confrontar las experiencias traumáticas, como impotencia, vergüenza, rabia, culpa y desesperanza, entre otras. Estos sentimientos son esperables en el marco del TEPT y es útil socializar al paciente a los elementos normativos de la psicopatología posttraumática. Es importante notar la diferencia entre normalizar sentimientos que se producen por el TEPT, por un lado, y normalizar el TEPT mismo, por el otro. El terapeuta debe proveer psicoeducación adecuada e indicar que el TEPT es un trastorno psiquiátrico que requiere tratamiento.

\section{Aspectos del Encuadre}

Para maximizar la posibilidad de que el tratamiento tenga un impacto positivo en el paciente, es necesario prestar especial atención al encuadre con que se realiza la psicoterapia. Se recomienda considerar lo siguiente:

- Usualmente la terapia centrada en el trauma se realiza una vez a la semana, o cada dos semanas, por un total de al menos de 10 sesiones (Kar, 2011) de 45 a 60 minutos. Se recomiendan sesiones semanales de 90 minutos para aquellas que incluyen técnicas de exposición, tanto en la imaginación como en vivo.

- Animar a aquellas personas con TEPT a que accedan al tratamiento tan pronto como sea posible. Igualmente, es importante enfatizar en las personas que han experimentado el trauma hace tiempo que el tratamiento puede ser efectivo, a pesar de la duración de la enfermedad.

- Luego de un desastre natural, es frecuente que el tratamiento de TEPT sea desarrollado en escenarios donde hay exposición a rastros evidentes del desastre. Es importante proveer un encuadre estable y proteger al paciente de riesgos reales. De no estar dadas las condiciones básicas de seguridad y estabilidad, es necesario evaluar la indicación al tratamiento de TEPT y potencialmente postergar el inicio de la intervención. Las condiciones del encuadre deben garantizar privacidad y confidencialidad. 


\section{¿Qué Otras Estrategias se Deben Considerar en una Intervención de TEPT?}

Además de las recomendaciones anteriores, las guías clínicas y los expertos sugieren estrategias adicionales que benefician la psicoterapia de pacientes con TEPT. Estas estrategias incluyen:

- Socializar frecuentemente al paciente con el TEPT y generar esperanza relativa al pronóstico en relación a los tratamientos disponibles. Esto ayuda a que el paciente comprenda que ha desarrollado un cuadro clínico, que tiene cierta frecuencia normativa y que existen buenos tratamientos disponibles.

- Incluir a los miembros de la familia en la psicoeducación y la planificación del tratamiento. Se deben considerar las necesidades y posibilidades que tiene la familia de brindar apoyo al paciente, junto con las necesidades de la persona con TEPT.

- Considerar las diferencias individuales (personalidad e historia), el ciclo vital, la identidad cultural, las respuestas de los familiares y amigos, y las creencias, pues estas influyen en cómo se lidia con la experiencia traumática, y las barreras para adherir al tratamiento y la recuperación (Cook \& Newman, 2014).

- Facilitar la seguridad psicológica y física mediante una alianza terapéutica basada en la confianza y el apoyo emocional (Cook \& Newman, 2014). Debe tomarse el tiempo necesario en estadios tempranos para explicar claramente la naturaleza y resultados esperados del tratamiento, generando una aproximación optimista (pero realista) y colaborativa.

- Anticipar aumentos transitorios en la sintomatología del cuadro. Esto es especialmente relevante en los tratamientos por exposición, en los que se puede notar al comienzo un aumento, por ejemplo, de la sintomatología de re-experimentación (e.g., pesadillas y flashbacks).

- Preguntar respecto del trauma, ya que muchas veces los afectados no reportan espontáneamente, porque no reconocen los efectos que tiene en sus vidas o los minimizan (Cook \& Newman, 2014). El paciente debe producir una narrativa sobre su experiencia traumática, la cual en el transcurso del tratamiento será cada vez más y más detallada y realista.

- Producir narraciones vívidas: pedirle al paciente que "coloree" con el mayor detalle sus narraciones. En general, es recomendable que los pacientes narren su trauma en tiempo presente y cerrando los ojos.

- Monitorear sistemáticamente la angustia del paciente cada cinco minutos durante el relato, utilizando un sistema de "anclas": "0 es nada de angustia, 100 es la angustia que usted sentiría si se encontrara atado a la línea del tren y ve a un tren que se avecina". Permitir que el paciente se recupere antes de terminar la sesión.

- Contrarrestar la impotencia de los afectados, promoviendo la sensación de logro en el paciente, involucrándolo activamente en el tratamiento y la toma de decisiones. Para esto es muy útil monitorear cuantitativamente la mejora del paciente y verificar disminuciones en conductas de evitación.

- Es probable que las primeras en pedir ayuda sean aquellas personas previamente traumatizadas. Es por esto que quienes prestan los servicios de salud mental deben poder lidiar con un amplio rango de eventos traumáticos.

\section{Atención de Pacientes con Comorbilidad}

Es común que, además del TEPT, los pacientes presenten al mismo tiempo otro trastorno psiquiátrico. Por ello, es importante considerar las siguientes recomendaciones en relación a las comorbilidades más frecuentes:

- Depresión y TEPT: en la mayoría de los casos TEPT debería ser tratado primero, ya que la depresión a menudo mejora a medida que los síntomas de TEPT se reducen. Sin embargo, los síntomas de la depresión necesitan ser manejados primero cuando impiden el compromiso efectivo en terapia o están asociados con un alto riesgo de suicidio. Es importante evaluar regularmente el riesgo suicida.

- Abuso de sustancias y TEPT: el tratamiento debería comenzar sobre las dos condiciones de forma simultánea, ya que las dos interactúan manteniéndose mutuamente y el tratamiento suele ser menos efectivo si una de ellas permanece sin tratarse. El tratamiento simultáneo de abuso de sustancias y TEPT parece acelerar la recuperación de ambos cuadros y disminuir las recaídas (Foa et al., 2013). Por otro lado, una terapia centrada en el trauma o de exposición prolongada podría aumentar el deseo y consumo de sustancias (Hien, Cohen, Miele, Litt \& Capstick, 2004; Solomon, Gerrity \& Muff, 1992) como estrategias de evitación. En el caso de que se decida comenzar primero con el tratamiento para abuso de 
sustancia, se debe tener presente que los síntomas TEPT pueden empeorar en la presencia de un síndrome de abstinencia aguda. Para ello se deben incluir estrategias acerca de cómo lidiar con el TEPT mientras la persona controla su abuso de sustancia. Es esencial evitar el consumo antes y después de los ejercicios de exposición en la imaginación y en vivo. Dependiendo del foco de atención clínica, terapeuta y paciente pueden acordar ciertos parámetros para que el consumo no afecte el circuito exposiciónhabituación.

- Duelo complicado y TEPT: A pesar de que el duelo produce un estrés severo, típicamente se resuelve solo y no necesita intervención clínica. Sin embargo, si el duelo se convierte en una condición debilitante crónica, se le llamada duelo complicado. Cuando los desastres de origen natural causan la muerte de muchas personas, es importante estar atentos a la presencia de un duelo complicado (Shear et al., 2011), que puede ocultarse tras la sintomatología de TEPT. De ser así, es necesario trabajar con la interacción entre las memorias traumáticas y el duelo complicado. Se recomienda el tratamiento especializado complicated grief treatment (Shear et al., 2011), el cual incluye técnicas similares a la exposición prolongada, además de enfocarse en los objetivos del paciente y sus relaciones interpersonales.

\section{¿Es Recomendable el Uso de Psicofármacos en Pacientes con TEPT?}

Usualmente no se utilizan psicofármacos como primera opción de tratamiento, sino que en combinación con el tratamiento psicológico como coadyuvantes bajo determinadas circunstancias. Ciertas consideraciones deben tomarse en cuenta para decidir incluir medicación:

- Los psicofármacos no deben ser usados dentro de las cuatro semanas de aparición de síntomas, a menos que la severidad del malestar no pueda ser manejada con psicoterapia o el paciente presente un severo deterioro psicosocial.

- Los psicofármacos deberán ser considerados cuando la persona no está obteniendo suficiente beneficio de la intervención psicológica por sí sola, no está dispuesta a participar en psicoterapia, no tiene acceso a una terapia centrada en el trauma o presenta problemas de salud mental adicionales, tales como depresión.

- Los psicofármacos más recomendados para el tratamiento de TEPT en adultos son los antidepresivos inhibidores selectivos para la recaptación de serotonina. Es relevante destacar que no se recomienda el uso de benzodiacepinas y antipsicóticos atípicos como monoterapia, ya que algunos estudios han concluido que estos medicamentos no muestran ser efectivos y que, incluso, podrían ser contraindicados. En específico, las benzodiacepinas han mostrado interferir con la extinción del miedo en terapias de exposición prolongada (Jeffreys, Capehart \& Friedman, 2012).

- Psicólogos y psiquiatras deben trabajar juntos para promover los resultados psicológicos y funcionales óptimos.

\section{¿Qué Evitar en una Psicoterapia Enfocada en Trauma?}

Si bien, como mostramos previamente, existen múltiples recomendaciones sobre qué hacer con un paciente con TEPT, también es importante considerar intervenciones posiblemente iatrogénicas que se deben evitar:

- No se debe forzar a los pacientes a hablar del trauma (Figueroa, Marín \& González, 2010). A veces los pacientes no recuerdan bien los eventos que causaron el trauma o no están preparados emocionalmente para enfrentarlos. Socializar al paciente respecto de los principios de acción del tratamiento es muy importante para ayudar al paciente a examinar sus memorias traumáticas y sus estados afectivos.

- Evitar las intervenciones de una sola sesión que se realizan en la fase aguda de los síntomas, como el debriefing (ventilación psicológica). La evidencia muestra que estos tratamientos no solo no tienen ninguna eficacia en la reducción de síntomas de TEPT y otros síntomas relacionados con el trauma, sino que además podrían tener un efecto perjudicial, incluso aumentando la incidencia de TEPT (van Emmerik, Kamphuis, Hulsbosch \& Emmelkamp, 2002).

- No se debe descuidar el funcionamiento psicológico y social global del paciente (inactividad social, falta de empleo, problemas familiares, entre otros). A su vez, el terapeuta debería evaluar desde el inicio las necesidades de apoyo laboral, social y práctico para proveer educación, apoyo y derivaciones adecuadas. En el caso de iniciar al paciente en un protocolo de exposición (ya sea PE o EMDR), es necesario focalizar 
el trabajo en estas técnicas y en la respuesta del paciente, ya que un desplazamiento intermitente del trabajo hacia otros focos podría resultar en un retraso de la curva de habituación.

Para ejemplificar algunas de las recomendaciones presentadas, en la Tabla 4 presentamos la segunda viñeta, que describe el caso ficticio de un paciente que recibe tratamiento especializado para TEPT. Además de describir la técnica de exposición prolongada, relatamos posibles dificultades que se presentaron en el tratamiento y que llevan al paciente a abandonarlo.

Tabla 4

Viñeta 2: Riesgos del Abandono

Alfredo recibe luego del terremoto de 2010 a Javier, un paciente diagnosticado adecuadamente con TEPT. Decide realizar con él un tratamiento de exposición prolongada, por lo que comienza explicándole en qué consiste esta terapia y, luego de algunas sesiones de entrevista, solicita a Javier narrar durante la sesión lo que le pasó durante el terremoto, detallando lo más posible (le pide cerrar los ojos y narrar en tiempo presente) toda su experiencia. Alfredo también le pide a Javier que cada cinco minutos monitoree sus niveles de ansiedad en una escala de 0 a 100.

Alfredo graba este relato que dura aproximadamente 30 minutos, para luego encomendar como tarea que Javier escuche esta grabación todos los días entre sesiones, preocupándose de registrar sus niveles de ansiedad cada cinco minutos.

Durante tres semanas, Javier asiste a sus sesiones con Alfredo donde narra y re-narra su trauma y graba las narraciones para ser escuchadas en su casa. Sin embargo, Javier no experimenta una disminución de sus síntomas y, por lo contrario, siente cada día mayor ansiedad y presenta una mayor cantidad de pesadillas y flashbacks de los que presentaba antes de asistir a terapia. Javier decide abandonar la terapia porque "he ido a varias sesiones y me siento peor que antes, creo que no hemos avanzado y que he desperdiciado mi tiempo y dinero".

¿Qué puede haber ocurrido en este caso?

1. La narración de la vivencia de Javier no es la más eficaz para producir exposición. Se debe volver, entonces, a identificar qué parte de la narración del suceso es la que genera mayores montos de angustia.

2. Alfredo no sociabilizó (y re-sociabilizó) a Javier lo suficiente con la terapia de exposición (por ejemplo, prescribir un aumento de los síntomas al principio del tratamiento), por lo que, al exponerse a síntomas más intensos de los que ya presentaba, pensó que la terapia no funcionaba y decide abandonar el tratamiento.

3. Javier luego de escuchar las grabaciones, y con la finalidad de disminuir su angustia, comenzó a tomar alcohol en dosis bajas para disminuir la ansiedad, cuestión que no fue monitoreada por Alfredo. Esto podría considerarse como una conducta evitativa de la exposición, por lo que se dificulta la habituación del paciente a su angustia.

¿Cuáles son los riesgos de finalizar una terapia de exposición antes de tiempo?

Uno de los riesgos de interrumpir una terapia de exposición antes de que se produzca una habituación a las memorias del trauma es que, si se ha producido una intensificación de los síntomas, por ejemplo, de la ansiedad, ese nivel exacerbado de ansiedad se estabiliza y la próxima vez que el paciente sufra ansiedad producto de una evocación del trauma será a partir de este nivel exacerbado. En otras palabras, se corre el riesgo de que el paciente que abandona presente síntomas de mayor intensidad que cuando inició el tratamiento.

\section{Auto-Cuidado de los Profesionales de Salud}

Trabajar con pacientes con TEPT puede ser intenso emocionalmente para los profesionales de salud, los cuales deben preocuparse de su auto-cuidado. Para esto se recomienda: 
- No exponerse a situaciones de sufrimiento, dolor y trauma que puedan estar más allá de sus capacidades de tolerancia personal (fatiga por compasión y trauma vicario).

- Prestar especial atención al desarrollo de competencias y habilidades, incluyendo supervisión regular o reuniones clínicas, establecer y mantener límites emocionales adecuados con los pacientes y responder pronto a las señales de estrés.

- Evitar el aislamiento. La responsabilidad de auto-cuidado debe ser compartida entre el profesional y su organización. Aquellos psicoterapeutas trabajando en localidades aisladas pueden beneficiarse del uso de internet para obtener supervisión y apoyo.

\section{Conclusiones}

A modo de conclusión, nos gustaría hacer un llamado a que los profesionales de salud mental en Chile se capaciten para poder brindar adecuadamente psicoterapia centrada en el trauma. A su vez, hacemos un llamado a que los programas educacionales de salud mental, tanto de pregrado como de postgrado, incluyan capacitación en TEPT y su tratamiento. Los desastres naturales son frecuentes en nuestro país y necesitamos estar preparados para brindar tratamiento especializado. Para quienes aún no reciben esta capacitación especializada, esperamos que las orientaciones presentadas en este artículo sirvan como una guía inicial. Las orientaciones fueron generadas a partir de una revisión no sistemática de las guías internacionales disponibles, revisión de artículos científicos y la opinión de expertos nacionales. El foco de estas orientaciones se centra en ayudar al paciente a disminuir su reactividad a las memorias traumáticas, procesar las memorias traumáticas evitando las distorsiones cognitivas y eliminar las conductas de evitación. Para lograr lo anterior, hemos descrito brevemente las narraciones como forma de exposición, modificar las distorsiones cognitivas, elaborar los sentimientos relacionados con el trauma, proporcionar psicoeducación y generar esperanza. A su vez, hemos destacado la importancia de proveer un encuadre estable y seguro, considerar comorbilidades, evitar ciertas intervenciones y la importancia del auto-cuidado del profesional.

\section{Referencias}

American Psychiatric Association (2013). Diagnostic and statistical manual of mental disorders (DSM-5) (5a ed.). Washington, DC: Autor. Australian Centre for Posttraumatic Mental Health (2013). Australian guidelines for the treatment of acute stress disorder \& posttraumatic stress disorder. Melbourne, Australia: Autor.

Breslau, N., Davis, G. C., Andreski, P. \& Peterson, E. (1991). Traumatic events and posttraumatic stress disorder in an urban population of young adults. Archives of General Psychiatry, 48, 216-222. https://doi.org/10.1001/archpsyc.1991.01810270028003

Bonanno, G. A., Galea, S., Bucciarelli, A. \& Vlahov, D. (2007). What predicts psychological resilience after disaster? The role of demographics, resources, and life stress. Journal of Consulting and Clinical Psychology, 75, 671-682. https://doi.org/10.1037/0022006X.75.5.671

Centro Sismológico Nacional Universidad de Chile (2016). Grandes terremotos en Chile: sismos importantes y/o destructivos (1570 a la fecha). Magnitud Ms mayor o igual a 7.0. Santiago, Chile: Autor. Extraído de http://www.csn.uchile.cl/sismologia/grandesterremotos-en-chile/

Chile, Ministerio de Planificación (2010). Plan de reconstrucción terremoto y maremoto del 27 de febrero de 2010: resumen ejecutivo. Concepción, Chile: Autor. Extraído de http://www.ministeriodesarrollosocial.gob.cl/pdf/plan-reconstruccion-resumen-ejecutivo.pdf

Chile, Ministerio de Salud (2010). Guía de vigilancia epidemiológica en emergencias y desastres. Santiago, Chile: Autor, Subsecretaría de Salud Pública. Extraído de https://issuu.com/preppers/docs/guia_de_vigilancia_epidemiologica_en_emergencias_y

Chile, Ministerio de Salud (2012). Manual para la protección y cuidado de la salud mental en situaciones de emergencias y desastres. Santiago, Chile: Autor. Extraído de http://www.cridlac.org/digitalizacion/pdf/spa/doc19524/doc19524-contenido.pdf

Chile, Oficina Nacional de Emergencia del Ministerio del Interior y Seguridad Pública (2018). Alertas. Extraído de http://www.onemi.cl/alertas/

Cook, J. M. \& Newman, E. (2014). A consensus statement on trauma mental health: The New Haven Competency Conference process and major findings. Psychological Trauma: Theory, Research, Practice, and Policy, 6, 300-307. https://doi.org/10.1037/a0036747

Courtois, C. A. \& Gold, S. N. (2009). The need for inclusion of psychological trauma in the professional curriculum: A call to action. Psychological Trauma: Theory, Research, Practice, and Policy, 1, 3-23. https://doi.org/10.1037/a0015224

Encinas, D. \& Valdés, G. (2012). Adaptación de un procedimiento psicoterapéutico para el estrés post-desastre aplicado después del terremoto y tsunami del 27 de Febrero de 2010 (Memoria de Grado no publicada), Facultad de Psicología, Universidad de Talca, Chile.

Figueroa, R. A., Marín, H. \& González, M. (2010). Apoyo psicológico en desastres: propuesta de un modelo de atención basado en revisiones sistemáticas y metaanálisis. Revista Médica de Chile, 138, 143-151. https://doi.org/10.4067/S0034-98872010000200001

Foa, E. B., Hembree, E. A. \& Rothbaum, B. O. (2007). Prolonged exposure therapy for PTSD: Emotional processing of traumatic experiences. Therapist guide. New York, NY: Oxford University Press.

Foa, E. B., Keane, T. M., Friedman, M. J. \& Cohen, J. A. (Eds.) (2009). Effective treatments for PTSD: Practice guidelines from the International Society for Traumatic Stress Studies (2a ed.). New York, NY: Guilford Press. 
Foa, E. B., Yusko, D. A., McLean, C. P, Suvak, M. K., Bux Jr., D. A., Oslin, D. ... Volpicelli, J. (2013). Concurrent naltrexone and prolonged exposure therapy for patients with comorbid alcohol dependence and PTSD: A randomized clinical trial. JAMA: The Journal of the American Medical Association, 310, 488-495. https://doi.org/10.1001/jama.2013.8268

Forbes, D., Creamer, M., Bisson, J. I., Cohen. J. A., Crow, B. E., Foa, E. B. ... Ursano, R. J. (2010). A guide to guidelines for the treatment of PTSD and related conditions. Journal of Traumatic Stress, 23, 537-552. https://doi.org/10.1002/jts.20565

Franklin, C. L, Sheeran, T. \& Zimmerman, M. (2002). Screening for trauma histories, posttraumatic stress disorders (PTSD) and subthreshold PTSD in psychiatric outpatients. Psychological Assessment, 14, 467-471. https://doi.org/0.1037//1040-3590.14.4.467

Galatzer-Levy, I. R. \& Bryant, R. A. (2013). 636,120 ways to have posttraumatic stress disorder. Perspectives on Psychological Science, 8, 651-662. https://doi.org/10.1177/1745691613504115

Garfin, D. R., Silver, R. C., Ugalde, F. J., Linn, H. \& Inostroza, M. (2014). Exposure to rapid succession disasters: A study of residents at the epicenter of the Chilean Bío Bío earthquake. Journal of Abnormal Psychology, 123, 545-556. https://doi.org/10.1037/a0037374

Goldmann, E. \& Galea, S. (2014). Mental health consequences of disasters. Annual Review of Public Health, 35, $169-183$. https://doi.org/10.1146/annurev-publhealth-032013-182435

Hamblen, J. L., Gibson, L. E., Mueser, K. T. \& Norris, F. H. (2006). Cognitive behavioral therapy for prolonged postdisaster distress. Journal of Clinical Psychology, 62, 1043-1052. https://doi.org/10.1002/jclp.20288

Hamblen, J. L., Norris, F. H., Pietruszkiewicz, S., Gibson, L. E., Naturale, A. \& Louis, C. (2009). Cognitive behavioral therapy for postdisaster distress: A community based treatment program for survivors of hurricane Katrina. Administration and Policy in Mental Health and Mental Health Services Research, 36, 206-214. https://doi.org/10.1007/s10488-009-0213-3

Hien, D. A., Cohen, L. R., Miele, G. M., Litt, L. C. \& Capstick, C. (2004). Promising treatments for women with comorbid PTSD and substance use disorders. The American Journal of Psychiatry, 161, 1426-1432. https://doi.org/10.1176/appi.ajp.161.8.1426

Inter-Agency Standing Committee (2007). IASC guidelines on mental health and psychosocial support in emergency settings. Genève, Suiza: Autor. Extraído de http://www.who.int/mental_health/emergencies/IASC_guidelines.pdf

Jeffreys, M., Capehart, B. \& Friedman, F. J. (2012). Pharmacotherapy for posttraumatic stress disorder: Review with clinical applications. JRRD: Journal of Rehabilitation Research \& Development, 49, 703-716. https://doi.org/10.1682/JRRD.2011.09.0183

Kar, N. (2011). Cognitive behavioral therapy for the treatment of post-traumatic stress disorder: A review. Neuropsychiatric Disease and Treatment, 7, 167-181. https://doi.org/10.2147/NDT.S10389

Kelly, K. A., Rizvi, S. L., Monson, C. M. \& Resick, P. A. (2009). The impact of sudden gains in cognitive behavioral therapy for posttraumatic stress disorder. Journal of Traumatic Stress, 22, 287-293. https://doi.org/10.1002/jts.20427

Kessler, R. C., Berglund, P., Demler, O., Jin, R., Merikangas, K. R. \& Walters, E. E. (2005). Lifetime prevalence and age-of-onset distributions of DSM-IV disorders in the National Comorbidity Survey replication. Archives of General Psychiatry, 62, 593-602. https://doi.org/10.1001/archpsyc.62.6.593

Kessler, R. C., Sonnega, A., Bromet, E., Hughes, M. \& Nelson, C. B. (1995). Posttraumatic stress disorder in the National Comorbidity Survey. Archives of General Psychiatry, 52, 1048-1060. https://doi.org/10.1001/archpsyc.1995.03950240066012

Leiva-Bianchi, M. \& Araneda, A. (2013). Prevalencia y sintomatología del estrés post traumático en personas que experimentan un terremoto y un tsunami. Salud \& Sociedad, 4, 146-155. https://doi.org/10.22199/S07187475.2013.0002.00003

Leiva-Bianchi, M., Cornejo, F., Fresno, A., Rojas, C. \& Serrano, C. (2018). Effectiveness of cognitive-behavioural therapy for post-disaster distress in post-traumatic stress symptoms after Chilean earthquake and tsunami. Gaceta Sanitaria, 32, $291-296$. https://doi.org/10.1016/j.gaceta.2017.07.018

Neria, Y., Nandi, A. \& Galea, S. (2008). Post-traumatic stress disorder following disasters: A systematic review. Psychological Medicine, 38, 467-480. https://doi.org/10.1017/S0033291707001353

Organización de las Naciones Unidas (2004). Vivir con el riesgo: informe mundial sobre iniciativas para la reducción de desastres. Genève, Suiza: Autor, Secretaría Interinstitucional de la Estrategia Internacional para la Reducción de Desastres. Extraído de http://www.cridlac.org/digitalizacion/pdf/spa/doc16481/doc16481.htm

Organización Mundial de la Salud (1992). CIE 10: décima revisión de la clasificación internacional de las enfermedades. Trastornos mentales y del comportamiento. Madrid, España: Meditor.

Ozer, E. J., Best, S. R., Lipsey, T. L. \& Weiss, D. S. (2003). Predictors of posttraumatic stress disorder and symptoms in adults: A metaanalysis. Psychological Bulletin, 129, 52-73. https://doi.org/10.1037/0033-2909.129.1.52

Resick, P. A., Nishith, P., Weaver, T. L., Astin, M. C. \& Feuer, C. A. (2002). A comparison of cognitive-processing therapy with prolonged exposure and a waiting condition for the treatment of chronic posttraumatic stress disorder in female rape victims. Journal of Consulting and Clinical Psychology, 70, 867-879. https://doi.org/10.1037/0022-006X.70.4.867

Shapiro, F. (1989). Eye movement desensitization: A new treatment for post-traumatic stress disorder. Journal of Behavior Therapy and Experimental Psychiatry, 20, 211-217. https://doi.org/10.1016/0005-7916(89)90025-6

Shapiro, F. (1995). Eye movement desensitization and reprocessing: Basic principles, protocols, and procedures. New York, NY: Guilford Press.

Shear, M. K., Simon, N., Wall, M., Zisook, S., Neimeyer, R., Duan, N. ... Keshaviah, A. (2011). Complicated grief and related bereavement issues for DSM-5. Depression and Anxiety, 28, 103-117. https://doi.org/10.1002/da.20780

Solomon, S. D., Gerrity, A. T. \& Muff, A. M. (1992). Efficacy of treatments for posttraumatic stress disorder: An empirical review. JAMA: The Journal of the American Medical Association, 268, 633-638. https://doi.org/10.1001/jama.1992.03490050081031

U.S. Department of Health and Human Services (2003). Achieving the promise: Transforming mental health in America. Final report. Rockville, MD: Autor, President's New Freedom Commission. Extraído de http://govinfo.library.unt.edu/mentalhealthcommission/reports/FinalReport/downloads/downloads.html

U.S. Department of Veterans Affairs \& U.S. Department of Defense (2010). VA/DoD clinical practice guideline for management of posttraumatic stress. Washington, DC: Autores, The Management of Post-Traumatic Stress Working Group. Extraído de http://www.healthquality.va.gov/guidelines/MH/ptsd/cpg_PTSD-FULL-201011612.pdf

van Emmerik, A. A. P., Kamphuis, J. H., Hulsbosch, A. M. \& Emmelkamp, P. M. G. (2002). Single session debriefing after psychological trauma: A meta-analysis. The Lancet, 360, 766-771. https://doi.org/10.1016/S0140-6736(02)09897-5

World Health Organization, War Trauma Foundation \& World Vision International (2011). Psychological first aid: Guide for field workers. Genève, Suiza: World Health Organization. http://www.searo.who.int/srilanka/documents/psychological_first_aid_guide_for_field_workers.pdf?ua=1 
Zlotnick, C., Johnson, J., Kohn, R., Vicente, B., Rioseco, P. \& Saldivia, S. (2006). Epidemiology of trauma, post-traumatic stress disorder (PTSD) and co-morbid disorders in Chile. Psychological Medicine, 36, 1523-1533. https://doi.org/10.1017/S0033291706008282

Fecha de recepción: Mayo de 2017.

Fecha de aceptación: Mayo de 2018. 